\title{
Utility and delivery of behavioural interventions to prevent sexually transmitted infections
}

\author{
Sevgi 0 Aral
}

\section{Correspondence to}

Dr Sevgi 0 Aral, Associate Director for Science, Division of STD Prevention, The National Center for HIV/AIDS, Viral Hepatitis, STD and TB Prevention, Centers for Disease Control and Prevention (CDC) 1600 Clifton Road, Mailstop E-02, Atlanta, GA 30333, USA; soa1@cdc.gov

Accepted 19 July 2011

This paper is freely available online under the BMJ Journals unlocked scheme, see http://sti. bmi.com/site/about/unlocked. xhtml

\section{INTRODUCTION}

Over the past three decades, research focus on the development and evaluation of behavioural interventions aimed at reducing the spread of sexually transmitted infections (STIs) including HIV has expanded considerably. Individual, couple and group level interventions aimed at improving condom use, HIV testing, linkage to care and retention, medication adherence, partner counselling and referral services, and decreasing number of partners have been developed and evaluated for many subpopulations. ${ }^{1-5}$ The interventions have been evaluated among African-Americans, Asian and Pacific Islanders, drug users, heterosexual adults, high-risk youth, Hispanic populations, men who have sex with men, people living with HIV, STI clinic patients, and transgender populations. However, despite their availability, behavioural interventions are inadequately utilised, and evidence of their population level impact is lacking. In this paper we discuss issues related to the utility of behavioural interventions, including questions around feasibility of implementation, scale-up and maintenance, and suggest new directions for planning, implementation, evaluation and continuous improvement of social and behavioural interventions in the context of STI and HIV prevention programmes.

\section{EVALUATION OF EFFICACY AND ASSESSMENT OF BEHAVIOURS}

Most evidence on the efficacy of behavioural interventions is based on self-reported behaviour, and change in self-reported behaviour, as outcome. Assessment and reporting of sexual behaviour is subject to ascertainment and reporting bias. ${ }^{6}$ Such bias is particularly important in the context of behavioural intervention trials, because the intervention directly manipulates the desirability of certain reported behaviours, and subjects cannot be effectively blinded to the intervention. For behavioural intervention trials, STI and HIV incidence are the outcome measures of greatest interest. Unfortunately, trials that use STI and HIV incidence as outcome measure have not shown high levels of efficacy. Of the seven randomised controlled trials of behavioural interventions that measured HIV incidence as outcome, none were found to be efficacious. ${ }^{7}$ Of the 27 randomised controlled trials of behavioural interventions that measured STI incidence as outcome, 17 were found to be efficacious; however, the magnitude of intervention effect varied widely. ${ }^{8}$ None of the behavioural trials assessed sustainability of behaviour change over time.

\section{BEHAVIOURS AND BEHAVIOUR SYSTEMS}

Behavioural intervention trials that use self-reports of behaviour as outcome measure are more likely to demonstrate efficacy than those that use STI/HIV incidence as outcome measure. One reason for this discrepancy may be the focus on specific behaviours. Most studies of behavioural risk and behavioural interventions focus on specific behaviours such as condom use, sexual frequency, numbers of partners, age at sexual initiation, or choice of high/low-risk partners, and treat these behaviours as if they were autonomous and independent of each other. Some studies use composite behaviour scales; however, these tend to be simple additive measures. Careful observation and review of the literature suggest that such behaviours constitute interdependent components of complex behavioural systems and should be treated and assessed as such. Individuals who become monogamous may simultaneously stop using condoms; individuals who start using condoms may increase their numbers of partners; individuals who use microbicides may stop using condoms; individuals who initiate sex relatively late in life may accumulate new partners faster during their early years of sexual activity; individuals with high-risk partners may also engage in other high-risk activities such as substance use, unsafe sex, multiple partners and the like. Risk behaviours tend to cluster in population subgroups and during particular stages of life. Risk behaviours are related to each other, and the relationships among them are complex and may take many forms. Risk behaviours may be conditional-individuals may use condoms only with high-risk partners; they may clusterindividuals who engage in high-risk sexual practices such as anal intercourse may also have many partners, not use condoms, use drugs and alcohol, and have sex with other high-risk individuals; they may be compensatory-individuals with high-risk partners may use condoms and avoid high-risk practices such as anal sex. Moreover, the effects of a number of behaviours on STI/HIV incidence may be additive, conditional, synergistic or antagonistic. In addition, risk and preventive behaviours, and changes in them, interact with biomedical states and changes in such states. For example, individuals with HIV infection may be more (or less) likely to use condoms or avoid insertive anal intercourse. Similarly, individuals receiving antiretroviral therapy may be more or less likely to engage in risky behaviours. Behavioural assessments and interventions that do not consider specific behaviours to be inter-related components of complex behaviour systems may fail to describe and intervene in the behavioural picture as a whole and may 
be subject to unexpected outcomes and unintended consequences that originate in the interdependencies among behaviours and between behaviours and biomedical states.

\section{THE LIMITED RELATIVE IMPORTANCE OF BEHAVIOURS OF INDIVIDUALS}

Recent research has shown that individuals' own behaviours have only a limited effect on their chances of acquiring an STI including HIV. ${ }^{9}$ In the USA, while white Americans are at risk of acquiring STIs if they engage in risky behaviours, African-Americans are at risk of acquiring STIs even if they engage in normative behaviours. ${ }^{9}$ Individuals' risk of acquiring STIs is highly dependent on the epidemiological context, their position in the sexual networks they belong to, and, more directly, on the behaviours and infection status of their sex partners. A person's risk of acquiring an STI depends on his/her partners' non-monogamy. ${ }^{10}$ More importantly, at the population level, spread of STI may be determined by presence and size of subpopulations marked by mutual non-monogamy where both partners have other partners. ${ }^{10}$

Our current understanding is that partners' behaviours, structure of sexual networks, linkages among sexual networks, and individuals' position in a sexual network all exert considerable influence on individuals' risk of acquiring and transmitting STI and the rate at which STIs spread in populations. Such understanding points to the limited role that individuals' own behaviours play in determining their STI risk. Thus, behavioural interventions aimed at changing individual behaviours may also be limited in their potential effects on STI risk and population level STI spread.

\section{TYPES OF BEHAVIOURAL INTERVENTIONS: MAXIMISING ADHERENCE AND MINIMISING DISINHIBITION}

The utility of behavioural interventions depends, in part, on the type of intervention in question. Some behavioural interventions target large groups of individuals, such as sex and HIV education programmes delivered in schools ${ }^{11}$ or through the mass media; others focus on individuals and tend to be time and labour intensive. Many behavioural interventions focus on risk or preventive behaviours; others focus on health behaviours such as testing and treatment. Most behavioural interventions aim to prevent acquisition of STI/HIV by uninfected individuals; others aim to prevent transmission of infection by infected individuals. A focus on prevention of transmission allows resources and attention to be concentrated on a smaller number of individuals; however, interventions may be less effective in preventing transmission-people tend to be more motivated to protect themselves and less motivated to protect others.

The majority of available behavioural interventions were developed independently of other interventions, with the aim of changing risk behaviours. In the light of recent promising developments in biomedical prevention of human papillomavirus and HIV infection, it may be important to redirect, in part, behavioural research and programmatic activity. ${ }^{12}$ Maximising adherence to biomedical recommendations regarding vaccination, testing, and microbicide and antiretroviral use, and minimising disinhibition that may result from perceptions of decreased health risks may be crucial new behavioural prevention goals in the current decade. Prevention of transmission through effective implementation of biomedical interventions, in combination with safer sex strategies and through innovative interventions focused on sexual networks and mixing patterns, may be the most effective (and cost-effective) approach overall.

\section{CONSEOUENCES OF BEHAVIOURAL INTERVENTIONS: EFFICACY, EFFECTIVENESS AND IMPACT}

Currently, there is a gap between the development and evaluation of efficacious interventions to prevent STIs including HIV and the implementation of the correct mix of interventions, at the right scale, in populations in order to achieve populationlevel impact. ${ }^{13}$ Many factors contribute to differences between efficacy under ideal trial conditions and effectiveness under real-life conditions. These include the characteristics of the study populations, the studied doses, the intensities, durations and frequencies of interventions (medications), contextual effects that interact with the interventions, and the like. Evaluation of the impact of interventions at the population level is more complicated, particularly because many interventions are implemented simultaneously in most settings. According to a recent formulation of the effect of different types of public health interventions, behavioural interventions such as counselling may have the least population level impact of all public health interventions. ${ }^{14}$ Reasons for the limited public health impact of behavioural interventions may include failure to achieve the required level of coverage, failure to sustain the intervention for the required duration, failure to implement required booster doses of the intervention, and failure to reimplement the intervention at the required frequency. In particular, issues of scale-up to achieve the coverage required for population level impact, and the related issues of repeat implementation at a frequency dictated by new additions to target populations, are important. New additions to sexually active populations are continuous because of the developmental nature of sexual activity. Moreover, turnover in key populations such as sex workers and drug users tends to be remarkably high. Even where individuals remain in these subpopulations, they tend to change their geographic location. Such movement into and out of highrisk populations and geographic areas greatly increases the need for repeated implementation of interventions. Where repeated implementation fails, the coverage required for population level impact cannot be achieved. Unfortunately, quite often, the required coverage is not even known. Mathematical modelling exercises can provide important help in the determination of required levels of coverage for population impact.

\section{INTERVENTIONS AND INTERVENTION SYSTEMS}

There is a recent growing recognition that a single intervention will not adequately slow down HIV transmission and that a combination of HIV prevention strategies might optimise HIV prevention impact. ${ }^{15}$ Interventions implemented in a given population context constitute complex systems. Such interventions are inter-related and their effects may be synergistic, antagonistic, additive or conditional, as they affect the incidence of STI including HIV. ${ }^{16}$ Assuming that, at the population level, effects of multiple interventions will be synergistic, or at least additive, may be overly optimistic. As behavioural interventions are introduced as components of intervention systems in a population, it is important to carefully consider the specific potential interactions among component interventions and plan accordingly.

\section{THE PLACE FOR BEHAVIOURAL INTERVENTIONS: THE ROLE OF CONTEXT, RESOURCES, PERSONAL AND PUBLIC HEALTH}

Two main functions of public health research and public health programmes, including STI/HIV prevention programmes, are to: (a) make relevant knowledge and materials available to individuals so they can protect their personal health and avoid 
acquisition and transmission; (b) implement strategic interventions to prevent the spread of infection in populations. Informing the general population about the prevalence and demographic and geographic distribution of infections, the efficacy of condoms and other biomedical interventions such as microbicides and pre- and post-exposure prophylaxis, and the $R R$ of particular sexual practices, is an intervention aimed at enhancing knowledge, an important preventive function that all public health programmes should adopt. Implementation of interventions aimed at reducing infection incidence at the population level is more complicated. ${ }^{16}$ Strategic decisions on the behavioural interventions to implement, the target populations for each intervention, the coverage and duration of implementation, and the costs, cost-effectiveness and expected population impact are marked by many difficult trade-offs. A recent analysis of optimum resource allocation for HIV prevention in the USA suggests that prevention efforts should be focused on HIV-infected people. ${ }^{17}$ An earlier framework had proposed a hierarchical ranking of subpopulations for prevention efforts ranging from infected persons with high-risk transmission behaviours to uninfected persons with low-risk behaviours. ${ }^{16}$ In any particular setting, the optimum mix of interventions will depend on the epidemiological, social and economic context and the available financial, human, organisational and cultural resources.

Acknowledgements I would like to thank Patricia Jackson for her excellent help with the preparation of this article.

Disclaimer The findings and conclusions in this paper are those of the author and do not necessarily represent the views of the Centers for Disease Control and Prevention.

\section{Competing interests None.}

Contributors SOA planned the outline and wrote the article.

Provenance and peer review Commissioned; externally peer reviewed.

\section{REFERENCES}

1. Marks G, Gardner LI, Craw J, et al. Entry and retention in medical care among HIV-diagnosed persons: a meta-analysis. AIDS 2010;24:2665-78.
2. Crepaz N, Norn AS, Rama SM, et al: HIV/AIDS Prevention Research Synthesis Team. The efficacy of behavioral interventions in reducing HIV-risk sex behaviors and incident STD in African-American and Hispanic STD clinic patients in the United States: a meta-analytic review. Sex Transm Dis 2007;34:319-32

3. Crepaz N, Marshall KJ, Aupont LW, et al. The efficacy of HIV/STI behavioral interventions for African-American females in the United States: a meta-analysis. Am J Public Health 2009;99:2069-78. http://www.ncbi.nlm.nih.gov/pubmed/ 19762676.

4. Darbes L, Crepaz N, Lyles CM, et al. The efficacy of behavioral interventions in reducing HIV risk behaviors and incident sexually transmitted diseases in heterosexual African Americans. AIDS 2008;22:1177-94. http://www.ncbi.nlm.nih. gov/pubmed/18525264.

5. Crepaz N, Marks G, Liau A, et al; HIV/AIDS Prevention Research Synthesis (PRS) Team. Prevalence of unprotected anal intercourse among HIV-diagnosed men who have sex with men (MSM) in the United States: a meta-analysis. AIDS 2009:23:1617-29. http://www.ncbi.nlm.nih.gov/pubmed/19584704.

6. DiClemente RJ, Sales JM, Danner F, et al. Association between sexually transmitted diseases and young adults' self-reported abstinence. Pediatrics 2011:127:209-13.

7. Padian NS, McCoy SI, Balkus JE, et al. Weighing the gold in the gold standard: challenges in HIV prevention research. AIDS 2010;24:621-35.

8. Wetmore CM, Manhart LE, Wasserheit JN. Randomized controlled trials of interventions to prevent sexual transmitted infections: Learning from the past to plan for the future. Epidemiol Rev 2010;32:121-36.

9. Halfors DD, Iritani BJ, Miller WC, et al. Sexual and drug behavior patterns and HIV and STD racial disparities: The need for new directions. Am J Public Health 2007:97:125-32.

10. Aral SO, Leichliter JS. Non-monogamy: risk factor for STI transmission and acquisition and determinant of STI spread in populations. Sex Transm Infect 2010;86 (Suppl 3):iii29-36.

11. Kirby DB, Laris BA, Rolleri LA. Sex and HIV education programs: their impact on sexual behaviours of young people throughout the world. J Adolesc Health 2007:40:206-7.

12. Cates W Jr. After CAPRISA 004: time to re-evaluate the HIV lexicon. Lancet 2010:376:495-6.

13. Aral So, Blanchard J, Lipshutz J. STD/HIV prevention intervention: efficacy, effectiveness and population impact. Sex Transm Infect 2008;84(Suppl 2):ii1-3.

14. Frieden TR. A framework for public health action: the health impact pyramid. Am J Public Health 2010;100:590-5

15. Kurth AE, Celum C, Baeten JM, et al. Combination HIV prevention: significance, challenges, and opportunities. Curr HIV/AIDS Rep 2011;8:62-72.

16. Aral So, Douglas JM Jr, Lipshutz JA, eds. Behavioral Interventions for Prevention and Control of Sexually Transmitted Diseases. New York: Springer Science+ Business Media, LLC, 2007:577.

17. Lasry A, Sansom SL, Hicks KA, et al. A model for allocating CDC's HIV prevention resources in the United States. Health Care Manag Sci 2011:14:115-24. 